\section{Utilidad de la determinación de la concentración sanguínea de rifampicina en pacientes con respuesta lenta al tratamiento antituberculoso}

La tuberculosis (TBC) se cura fácilmente cuando se administra el tratamiento adecuado en el momento oportuno. En ausencia de resistencia a los fármacos antituberculosos, los tratamientos breves habituales proporcionan tasas de curación $>95 \%$. Los fracasos son atribuibles al retraso de la detección y tratamiento de la enfermedad, al incumplimiento del tratamiento, a la resistencia a múltiples fármacos, a la infección por el virus de la inmunodeficiencia humana $(\mathrm{VIH})$ y a otras enfermedades concomitantes. Los pacientes sin estos factores de riesgo suelen responder bien al tratamiento. No obstante, ha habido informes recientes de casos que, sin presentar ninguno de estos factores de riesgo, responden mal al tratamiento, hecho atribuido a la presencia de concentraciones subterapéuticas de los fármacos antituberculosos.

El objetivo del presente estudio consistió en investigar el papel de las concentraciones subterapéuticas de rifampicina (RIF) en la respuesta lenta al tratamiento antituberculoso, definida como la ausencia de mejoría clínica y radiográfica y la presencia continuada de bacilos en el esputo al cabo de 3 meses de tratamiento antituberculoso apropiado.

Los pacientes elegibles para este estudio fueron todos los adultos con TBC pulmonar con baciloscopia y cultivo de esputo positivos para bacilos no resistentes a los fármacos antituberculosos registrados entre agosto de 1997 y agosto de 1999 en los departamentos de salud de los condados de Shelby y Washington del estado de Tennessee (Estados Unidos). Todos ellos recibieron inicialmente tratamiento antituberculoso con observación directa (DOT), consistente en la administración oral de cuatro fármacos: RIF, $600 \mathrm{mg} /$ día; isoniazida (INH), $300 \mathrm{mg} /$ día; pirazinamida, $25 \mathrm{mg} / \mathrm{kg} /$ día, y etambutol, $25 \mathrm{mg} / \mathrm{kg} /$ día. Los cuatro fármacos se administraron durante 2 meses, tras los cuales los pacientes siguieron recibiendo únicamente INH y RIF durante otros 4 meses o hasta que los frotis y cultivos del esputo dieran negativo en dos ocasiones consecutivas.

Los pacientes fueron examinados mensualmente por un médico y en cada consulta se les realizaron exámenes oculares, radiografías de tórax, pruebas funcionales hepáticas, determinaciones de la concentración de albúmina, baciloscopias y cultivos de esputo. En aquellos con respuesta lenta al tratamiento antituberculoso se procedió a determinar las concentraciones sanguíneas de RIF por cromatografía de líquidos de alto rendimiento. Se consideraron terapéuticas las concentraciones de RIF entre 8 y $24 \mu \mathrm{g} / \mathrm{mL}$. A los pacientes con concentraciones subterapéuticas se les aumentó la dosis de RIF a 900 mg y después se volvió a determinar la concentración sérica del fármaco. Si seguía siendo inferior al intervalo terapéutico se volvió a aumentar la dosis de RIF y se repitió una vez más la determinación de su concentración sérica. Para identificar otras enfermedades o medicaciones que pudieran alterar la farmacocinética de la RIF, se revisaron las historias clínicas de los pacientes y se les interrogó sobre la toma de otras medicaciones, incluidas las hierbas, los remedios holísticos y fármacos que no necesitaran prescripción médica.

Durante los 2 años de estudio se inició un tratamiento antituberculoso en 124 nuevos casos de TBC pulmonar no resistente a los fármacos. Seis de ellos presentaron respuesta lenta al DOT: cinco hombres y una mujer con una media de 50,7 años. Tres presentaron Mantoux positivo. El intervalo medio entre el inicio del tratamiento y su clasificación como casos con respuesta lenta al DOT fue de 19 semanas. Los seis presentaban concentraciones subterapéuticas de RIF con la dosis inicial de 600 $\mathrm{mg}$; después de aumentarla a $900 \mathrm{mg}$ se alcanzaron concentraciones terapéuticas en cinco casos. En el otro, para alcanzar concentraciones terapéuticas fue necesario aumentar la dosis de RIF a $1500 \mathrm{mg}$. El intervalo medio entre el ajuste de la dosis y la manifestación de la respuesta clínica fue de 5,33 semanas.

Tres pacientes consumían cantidades moderadas de alcohol y uno estaba infectado por el VIH. Este último estaba siendo tratado con zidovudina, pero no con inhibidores de la proteasa, y con antibióticos profilácticos (difluconazol, azitromicina y trimetoprim/sulfametoxazol). Ninguno de los seis consumía remedios holísticos, hierbas ni medicamentos que no necesitaran receta médica, ninguno presentaba síntomas de malabsorción y todos tenían concentraciones séricas normales de albúmina.

Todos los pacientes completaron con éxito su tratamiento antituberculoso, sin que se registraran reacciones adversas causadas por el aumento de la dosis de RIF. Los bacilos tuberculosos aislados en los seis pacientes no mostraron resistencia a los fár- 
macos adquirida a lo largo del estudio ni cambios de la concentración mínima inhibidora de RIF. La TBC pulmonar no recidivó en ningún caso.

En opinión de los autores, este estudio indica que la determinación de las concentraciones séricas de RIF es una medida útil en el tratamiento de los pacientes con TBC pulmonar con respuesta lenta al tratamiento antituberculoso, aunque no estén infectados por el VIH. (Mehta JB, Shantaveerapa H, Byrd Jr RP, Morton SE, Fountain F, Roy T. Utility of rifampin blood levels in the treatment and follow-up of active pulmonary tuberculosis in patients who were slow to respond to routine directly observed therapy. Chest 2001;120:1520-1524.)

\section{Seguridad de la administración neonatal de la vacuna contra la hepatitis B}

En los Estados Unidos, una considerable proporción de niños reciben la vacuna contra la hepatitis B (HB) en el período neonatal inmediato. Hay datos anecdóticos que indican que la vacunación contra la HB en el período neonatal se asocia a un aumento de la incidencia de fiebre, y la fiebre en los primeros días de vida puede hacer necesaria la realización de pruebas diagnósticas para descartar la existencia de sepsis. Por consiguiente, si la vacunación contra la HB aumentara realmente la incidencia de fiebre, podría llevar a la realización de pruebas caras e innecesarias. El objetivo de este estudio consistió en investigar la relación entre la vacunación contra la HB y los episodios febriles y la consiguiente realización de estudios diagnósticos innecesarios en los lactantes vacunados.

Para ello se efectuó un estudio prospectivo en un centro médico de San Francisco en el que participaron los recién nacidos normales a término nacidos entre el 1 de noviembre de 1991 y el 30 de abril de 1994.

Las comparaciones de las tasas de acontecimientos adversos se realizaron con dos definiciones de los vacunados. En el primer análisis se consideraron vacunados los lactantes que habían recibido una dosis de la vacuna contra la HB en los primeros 21 días de vida; en el segundo solo se consideraron vacunados los que recibieron una dosis de la vacuna en los primeros 2 días de vida. Como la mayoría de los lactantes vacunados habían nacido después de 1992 y la mayoría de los no vacunados habían nacido antes de 1993, se realizó un análisis secundario de los episodios febriles en el que solo se incluyeron los niños nacidos después de octubre de 1992.

Las diferencias entre proporciones se analizaron con la prueba de la $\chi^{2}$ y las diferencias entre medias de variables continuas mediante análisis de la varianza. La fuerza de las asociaciones se estimó mediante el riesgo relativo (RR).

Durante el período de estudio nacieron 6521 niños, 866 de los cuales fueron excluidos por diferentes motivos. De los 5655 casos restantes, 3302 fueron vacunados en los primeros 21 días de vida y los otros 2353 , no. Entre los vacunados, 2718 (82\%) lo fueron en los primeros 2 días de vida.

Las tasas de episodios febriles, reacciones alérgicas, convulsiones y otras enfermedades neurológicas no presentaron diferencias entre los niños vacunados en los primeros 2 días de vida, los vacunados en los primeros 21 días y los no vacunados en las primeras 3 semanas. Los niños de los diferentes grupos con episodios febriles tampoco presentaron diferencias con respecto a la edad de aparición de la fiebre: media de 10,3 días en los no vacunados, 11,4 días en los vacunados en los días 0 y 1, y 14,0 días en los vacunados entre los días 2 y 21 ( $P=0,31$ mediante análisis de varianza).

En el análisis secundario de los niños nacidos después de octubre de 1992 participaron 3409 lactantes, de los cuales $2843(83,4 \%)$ fueron vacunados. El RR de fiebre entre los vacunados, en comparación con los no vacunados, fue de 0,94 (intervalo de confianza del 95\%: 0,77 a 1,15; $P=0,48$ ).

En los niños vacunados se realizaron menos cultivos de sangre y líquido cefalorraquídeo que en los no vacunados, cualquiera que fuera la definición de los vacunados. Además, la proporción de niños con cultivos positivos fue significativamente menor en los vacunados que en los no vacunados.

Así pues, este estudio no proporcionó pruebas de que la vacunación neonatal con la HB se asocie a un aumento del número de episodios febriles, de acontecimientos adversos de tipo alérgico o neurológico ni de investigaciones diagnósticas de sepsis neonatal. (Lewis E, Shinefield HR, Woodruff BA, Black SB, Destefano F, Chen RT, et al. Safety of neonatal hepatitis $B$ vaccine administration. Pediatr Infect Dis J 2001;20:1049-1054.)

\section{Brote de meningitis eosinofílica por Angiostrongylus cantonensis en viajeros regresados del Caribe}

Angiostrongylus cantonensis es la causa infecciosa más frecuente de meningitis eosinofílica en el ser humano. El huésped definitivo de este nemátodo es la rata y los humanos se infectan por la ingestión de larvas en estadio tres presentes en huéspedes intermedios (caracoles) o transportadores (camarones, peces) crudos o mal cocinados, o en productos frescos (lechugas) contaminados por estos huéspedes. Las larvas ingeridas penetran en los vasos intestinales y acaban llegando a las me- 
ninges, donde mueren al cabo de poco tiempo, produciendo una reacción eosinofílica que se manifiesta como meningitis aséptica. Aunque la infección es autolimitada en la mayoría de los casos, algunos sufren secuelas neurológicas y también se han descrito algunos casos mortales. La mayoría de los casos ocurren de forma aislada, pero también se han descrito algunos brotes. En este estudio se describe un brote de meningitis eosinofílica por A. cantonensis en un grupo de viajeros estadounidenses que habían estado en Jamaica.

A finales de abril del año 2000 ingresaron en un hospital de Chicago dos estudiantes de medicina que presentaban antecedentes de cefalea, cervicalgia y parestesias con agravación progresiva en los 10 días anteriores y que formaban parte de un grupo de 23 personas que habían estado una semana en Jamaica. El análisis del líquido cefalorraquídeo (LCR) fue compatible con meningitis aséptica y uno de los pacientes presentaba un 36\% de eosinófilos. Durante la semana y media siguiente ingresaron con meningitis otros siete viajeros del mismo grupo.

Los casos de meningitis eosinofílica se definieron clínicamente como una cefalea de inicio agudo en los 35 después del regreso de Jamaica, acompañada por al menos uno de los siguientes síntomas: trastornos visuales, fotofobia, rigidez de la nuca, cervicalgia, hiperestesias o parestesias. La eosinofilia se definió como la presencia de al menos un $10 \%$ de eosinófilos en el recuento total de leucocitos en el LCR o de al menos 600 eosinófilos $/ \mathrm{mm}^{3}$ en la sangre periférica.

Los análisis serológicos se efectuaron en dos muestras de sangre obtenidas durante la fase aguda de la enfermedad y durante la fase de convalecencia. Los anticuerpos frente al antígeno de $31 \mathrm{kDa}$ de A. cantonensis se detectaron mediante inmunoelectrotransferencia (Western blot). Para excluir otras posibles causas de meningitis eosinofílica también se usaron inmunoensayos enzimáticos para detectar anticuerpos frente a Strongyloides stercoralis, Toxocara spp y Trichinella spiralis.

Para identificar los factores de riesgo de meningitis eosinofílica se entrevistaron todos los viajeros en dos ocasiones, utilizando para ello cuestionarios estructurados que proporcionaban información sobre los síntomas y sobre exposiciones potencialmente relevantes, tanto en los Estados Unidos como en Jamaica y en los vuelos de ida y vuelta.

Doce de los 23 viajeros (52\%) cumplieron la definición de caso de meningitis eosinofílica y 9 de ellos fueron hospitalizados. El intervalo mediano entre la salida de Jamaica y el inicio de los síntomas fue de 11 días (6 a 31). Por definición, todos los pacientes tenían cefalea; los síntomas acompañantes más frecuentes fueron los trastornos visuales o fotofobia, la cervicalgia o rigidez de la nuca y la fatiga. Cinco pacientes dijeron haber tenido fiebre, pero durante la hospitalización solo se documentaron temperaturas $>37,8^{\circ} \mathrm{C}$ en dos casos. Se realizaron punciones lumbares en los nueve pacientes hospitalizados y en todos ellos acabó documentándose la presencia de eosinofilia, a pesar de que en el examen inicial solo cuatro (44\%) tenían eosinofilia en la sangre periférica, y cinco (56\%) en el LCR. En los siete casos en los que se efectuaron exámenes microscópicos no se identificaron larvas en el LCR. Los síntomas acabaron resolviéndose en los 12 pacientes, aunque en 8 de ellos (67\%) las cefaleas duraron al menos 4 semanas (hasta 6 a 8 semanas en dos casos). Un paciente todavía tiene un ligero temblor en reposo en los brazos y piernas 6 meses después del inicio de la enfermedad.

Se detectaron anticuerpos frente al antígeno de $31-\mathrm{kDa}$ de $A$. cantonensis en las muestras de suero de la fase aguda y de convalecencia de un paciente $(8 \%)$ y solo en las muestras de la fase de convalecencia de 10 pacientes (83\%), pero no en ninguno de los dos tipos de muestras de suero de ninguno de los otros 11 viajeros. En ninguno de los 21 casos en los que se efectuaron las pruebas correspondientes se detectaron signos serológicos de infección por T. spiralis o Toxocara spp. En dos casos se detectaron anticuerpos frente a $S$. stercoralis, en uno de ellos a concentraciones muy bajas.

De los siete restaurantes frecuentados por los viajeros, solo uno sirvió a todos los pacientes. También hubo una sola comida de ese restaurante que hubieran comido todos los pacientes; 12 de los 16 viajeros que la comieron enfermaron, mientras que entre los 7 que no la comieron no hubo ninguno que enfermara. Los 12 pacientes comieron ensalada, único plato que se asoció a un riesgo significativo de enfermedad (12 de los 13 que comieron ensalada enfermaron, frente a ninguno de los 3 que no la comieron). Ningún viajero había comido caracoles ni pescado o marisco crudo o mal cocido durante todo el viaje.

Aunque ninguno de los 12 casos descritos se confirmó parasitológicamente, los datos clínicos y serológicos proporcionan pruebas convincentes de que el agente etiológico de este brote de meningitis eosinofílica fue $A$. cantonensis. Los pacientes no fueron tratados con antihelmínticos debido al riesgo teórico de que este tratamiento exacerbe los síntomas neurológicos debido a la muerte de las larvas presentes en el sistema nervioso central. La mayoría de los casos respondieron al tratamiento conservador, consistente en punciones lumbares y corticoesteroides.

La presencia de cefalea, hipertensión intracraneana y pleocitosis, con o sin eosinofilia, en viajeros 
en riesgo debería despertar la sospecha de infección por $A$. cantonensis, especialmente si se acompaña de parestesias o hiperestesias. (Slom TJ, Cortese MM, Gerber SI, Jones RC, Holtz TH, Lopez AS, et al. An outbreak of eosinophilic meningitis caused by $\mathrm{An}$ giostrongylus cantonensis in travelers returning from the Caribbean. N Engl J Med 2002;346:668-675.)

\section{Toxoplasma gondii requiere la síntesis de novo de pirimidinas para ser virulento}

Toxoplasma gondii es un parásito intracelular obligado que puede producir malformaciones congénitas graves y encefalitis potencialmente mortal en pacientes inmunodeprimidos. El protozoo posee una vía fragmentada para rescatar las bases pirimidínicas derivadas del propio parásito o de la célula huésped, mediada exclusivamente por la uracilofosforibosiltransferasa. La interferencia en la función de esta enzima no afecta al crecimiento de los taquizoitos de T. gondii, lo cual indica que puede ser necesaria una vía de síntesis de novo de pirimidinas para que el parásito crezca.

Los autores de este informe analizaron la virulencia de mutantes de T. gondii carentes de carbamoil-fosfato-sintetasa II (auxotróficas para el uracilo) para determinar si dicha virulencia requiere la síntesis de novo de pirimidinas in vivo. Esta enzima CPSII es la enzima reguladora clave de la síntesis de novo de pirimidinas y presenta propiedades distintas de las de la CPSII de las células huésped de los mamíferos.

Se clonó un fragmento de ADN genómico CPSII HindIII de 6,6 kilobases (kb) de la cepa RH, que contiene exones que poseen una gran similitud con la CPSII de los hongos, plantas y mamíferos, y se alteró la única copia cromosómica de este fragmento para obtener las cepas cps1-1 y cps1-2, auxotróficas para el uracilo. Ninguna de estas dos cepas tenía actividad CPSII detectable, en comparación con la actividad detectada en la cepa salvaje RH. En ausencia de suplementos de uracilo el parásito invadía normalmente los tejidos, pero una vez dentro de las células era incapaz de replicarse. Con concentraciones de uracilo $<20 \mu \mathrm{M}$ prácticamente no había crecimiento.

Se comprobó que la alteración del gen CPSII era la lesión genética responsable del auxotrofismo para el uracilo de las cepas cps1-1 y cps2-1. La transfección de estas cepas con un fragmento CPSII HindIII de 6,6 kb de la cepa $\mathrm{RH}$ rescató un número significativo de parásitos que pudieron crecer normalmente en ausencia de uracilo.

La virulencia de los mutantes de $T$. gondii con el gen CPSII anulado se probó en un modelo de to- xoplasmosis letal en ratones BALB/c. La cepa RH es hipervirulenta en todas las cepas de ratones, con una dosis letal del $100 \%$ de aproximadamente 10 parásitos. Los ratones inyectados por vía intraperitoneal con una pequeña dosis de esta cepa mueren rápidamente (supervivencia mediana de 9 días). En cambio, los ratones inyectados con 103 a 107 taquizoitos de las cepas mutantes cps1-1 o cps2-1 sobrevivieron a la infección y 3 semanas después de la inoculación no se recuperaron parásitos de la cavidad peritoneal. Los ratones inoculados con estas cepas sobrevivieron más de 12 meses sin que se observaran signos de persistencia del parásito. En cambio, los parásitos rescatados por transfección de las cepas auxotróficas para el uracilo con el fragmento CPSII HindIII de $6,6 \mathrm{~kb}$ salvaje fueron altamente virulentos.

También se investigó la virulencia de la cepa cps1-1 en ratones BALB/c con el gen del interferón $\gamma($ IFN- $\gamma$ ) anulado. Como el IFN- $\gamma$ es necesario para que el huésped pueda controlar la infección por T. gondii, los ratones con este gen anulado sucumben rápidamente a la toxoplasmosis, incluso tras la infección con cepas normalmente avirulentas. Así pues, como era de esperar, estos ratones fallecieron rápidamente tras la infección con la cepa $\mathrm{RH}$ (supervivencia mediana de 9 días), pero, inesperadamente sobrevivieron más de 6 meses a la infección con inóculos de 103 a 107 taquizoitos de la cepa mutante cps1-1, sin mostrar signos de persistencia del parásito.

Por último, se investigó si los mutantes avirulentos auxotróficos para el uracilo podían proteger a los ratones frente a la infección letal por toxoplasma. Para ello se inyectaron taquizoitos de la cepa cps1-1 en ratones BALB/c y 40 días después se les inyectó una dosis letal de la cepa RH. Las dosis $>104$ taquizoitos de la cepa cps1-1 fueron altamente eficaces para inducir inmunidad protectora a largo plazo en los ratones BALB/c.

Los resultados de este estudio indican que T. gondii podría depender totalmente de su propia vía de síntesis de novo de pirimidinas in vivo. Por consiguiente, la identificación de compuestos que limiten la biosíntesis de novo de pirimidinas en T. gondii podría ser útil para desarrollar nuevos fármacos antiparasitarios. Los mutantes auxotróficos descritos en este estudio son notablemente avirulentos $\mathrm{y}$, además, pueden inducir inmunidad protectora a largo plazo frente a la toxoplasmosis, por lo que también resultan muy prometedores para el desarrollo de una vacuna frente a ella. (Fox BA, Bzik DJ. De novo pyrimidine biosynthesis is required for virulence of Toxoplasma gondii [Letter]. Nature 2002;415(6874): 926-929.) 


\section{La dieta complementa los fármacos hipolipidemiantes en varones hipercolesterolémicos}

Los inhibidores de la 3-hidroxi-3-metilglutaril coenzima A reductasa (HMG-CoA reductasa), conocidos también como estatinas, reducen la morbilidad y la mortalidad cardiovasculares en pacientes con cardiopatía coronaria y en varones sanos con alto riesgo de cardiopatía coronaria. El efecto hipocolesterolemiante de las estatinas es superior al de diferentes regímenes dietéticos y sus efectos favorables sobre la morbilidad y la mortalidad cardiovascular se han atribuido a la disminución del colesterol de las lipoproteínas de baja densidad (LDL-C) y de los triglicéridos, y al aumento del colesterol de las lipoproteínas de alta densidad (HDL-C). A su vez, los efectos de la dieta de tipo mediterráneo, con pocas grasas saturadas y rica en ácidos grasos omega-3, podrían ser mediados principalmente por factores distintos de los lípidos séricos.

Existe escasa información acerca de la interacción entre la dieta y las estatinas en lo que se refiere a sus efectos sobre los lípidos y las lipoproteínas séricas, la sensibilidad a la insulina y las concentraciones circulantes de vitaminas y provitaminas antioxidantes. Por consiguiente, el objetivo del presente estudio consistió en investigar los efectos de la dieta y de una estatina, la simvastatina, por separado y combinadas, sobre estos parámetros.

Para ello se realizó un ensayo clínico aleatorizado, controlado, de diseño cruzado, en 120 varones hipercolesterolémicos (colesterol sérico en ayunas $\geq 232 \mathrm{mg} / \mathrm{dL} ; 6,0 \mathrm{mmol} / \mathrm{L}$ ) de 35 a 64 años que no habían sido tratados con anterioridad, residentes en Turku, Finlandia. El estudio se realizó entre agosto de 1997 y junio de 1998. El tamaño de la muestra se calculó asumiendo que es posible detectar una diferencia de $15 \mathrm{mg} / \mathrm{dL}(0,4 \mathrm{mmol} / \mathrm{L})$ en las dos medidas de eficacia principales (el colesterol total y el LDL-C) con una potencia del $80 \%$ y un error de tipo I del 5\% $(n=88)$. Todos los participantes completaron el estudio.

Los criterios de inclusión fueron: colesterol sérico en ayunas entre 232 y $309 \mathrm{mg} / \mathrm{dL}$ ( 6,0 y 8,0 $\mathrm{mmol} / \mathrm{L})$ y triglicéridos séricos en ayunas $\leq 266$ $\mathrm{mg} / \mathrm{dL}(3,0 \mathrm{mmol} / \mathrm{L})$. Se excluyeron los individuos con índice de masa corporal $>32 \mathrm{~kg} / \mathrm{m}^{2}$, cardiopatía coronaria, enfermedad cerebrovascular, claudicación, o hipertensión, hiperlipidemia o diabetes en tratamiento farmacológico.

Tras una fase previa de 4 a 6 semanas de tratamiento con placebo, los participantes fueron asignados aleatoriamente a un grupo que recibió su dieta habitual $(n=60)$ o a un grupo que recibió tratamiento dietético $(n=60)$. A su vez, cada uno de estos grupos fue asignado, de forma aleatoria, do- blemente enmascarada y cruzada, para recibir simvastatina ( $20 \mathrm{mg} /$ día) o placebo durante 12 semanas cada uno ( $n=30$ por grupo). Los principales objetivos del tratamiento dietético consistieron en reducir la ingesta de calorías procedentes de las grasas saturadas y transinsaturadas a no más del $10 \%$, sustituyéndolas parcialmente por grasas monoinsaturadas y poliinsaturadas ricas en ácidos grasos omega-3; en mantener una ingesta de colesterol $\leq 250 \mathrm{mg} /$ día; en proporcionar una ingesta de ácidos grasos omega-3 de origen vegetal (ácido linolénico) y marino $\geq 4 \mathrm{~g} /$ día; en mantener una razón < 4:1 entre los ácidos grasos poliinsaturados omega- 6 y omega-3, y en aumentar la ingesta de frutas, vegetales y fibra soluble.

Las principales medidas de eficacia fueron los cambios de las concentraciones de colesterol total, LDL-C y HDL-C, triglicéridos, apolipoproteína B, insulina, glucosa y antioxidantes al cabo de las 12 semanas de cada período de tratamiento.

Hubo un buen cumplimiento del tratamiento farmacológico (91 a 95\%, según el grupo). En general, el tratamiento dietético permitió alcanzar los objetivos predeterminados y disminuyó el colesterol total en un $7,6 \%(P<0,001)$, el LDL-C en un $10,8 \%(P<0,001)$, el HDL-C en un $4,9 \%(P=0,01)$, la apolipoproteína $\mathrm{B}$ en un $5,7 \%(P=0,003)$, la insulina sérica en un $14,0 \%(P=0,02)$ y el $\alpha$-tocoferol en un $3,5 \%(P=0,04)$; las concentraciones de triglicéridos, apolipoproteína A1 y glucosa no sufrieron cambios.

A su vez, el tratamiento con simvastatina disminuyó el colesterol total en un 20,8\%, el LDL-C en un $29,7 \%$, los triglicéridos en un $13,6 \%$, la apolipoproteína B en un $22,4 \%$, el $\alpha$-tocoferol en un $16,2 \%$, el $\beta$-caroteno en un $19,5 \%$ y el ubiquinol-10 en un $22,0 \%(P<0,001$ para todos estos cambios), mientras que aumentó el HDL-C en un 7,0\% $(P<0,001)$, la insulina sérica en un $13,2 \%(P=0,005)$ y la apolipoproteína A1 en un 2,4\%. Las concentraciones de glucosa no cambiaron en ningún grupo. La tensión arterial no se vio afectada por la dieta ni por el tratamiento farmacológico. Los efectos de la dieta y de la simvastatina fueron independientes y aditivos.

Entre los resultados obtenidos, cabe destacar que los efectos de la dieta y de la simvastatina fueron aditivos (por ejemplo, las reducciones del LDL-C proporcionadas por la dieta, la simvastatina y la combinación de ambos tratamientos fueron del 11,30 y $41 \%$, respectivamente) y que la simvastatina redujo en un 16 a $22 \%$ las concentraciones séricas de tres importantes vitaminas y provitaminas antioxidantes: el $\alpha$-tocoferol, el $\beta$-caroteno y el ubiquinol-10.

El estudio no está exento de limitaciones, como la imposibilidad de administrar el tratamiento dietético de forma doblemente enmascarada. Para 
evitar los efectos de confusión generados por el ciclo menstrual, los anticonceptivos orales y el tratamiento de reposición de estrógenos, solo se incluyeron hombres y, por lo tanto, los resultados solo son aplicables al sexo masculino. También queda por confirmar la factibilidad de ambos tratamientos a largo plazo e investigar sus efectos sobre otros aspectos que no se abordaron en este estudio, tales como la agregación plaquetaria, la hemostasia, la fibrinólisis, la función endotelial y la tendencia a las arritmias.

Los autores concluyen que la combinación de una dieta de tipo mediterráneo con el tratamiento con una estatina en varones hipercolesterolémicos no diabéticos no solo produce un efecto beneficioso aditivo de reducción del colesterol total y del LDL-C, sino que también contrarresta el aumento de la insulinemia en ayunas asociado al tratamiento farmacológico. Estos resultados destacan la importancia de la dieta como parte integral del tratamiento con estatinas en pacientes hipercolesterolémicos. (Jula A, Marniemi J, Huupponen R, Virtanen A, Rastas M, Rönnemaa T. Effects of diet and simvastatin on serum lipids, insulin, and antioxidants in hypercholesterolemic men. A randomized controlled trial. JAMA 2002;287: 598-605.)

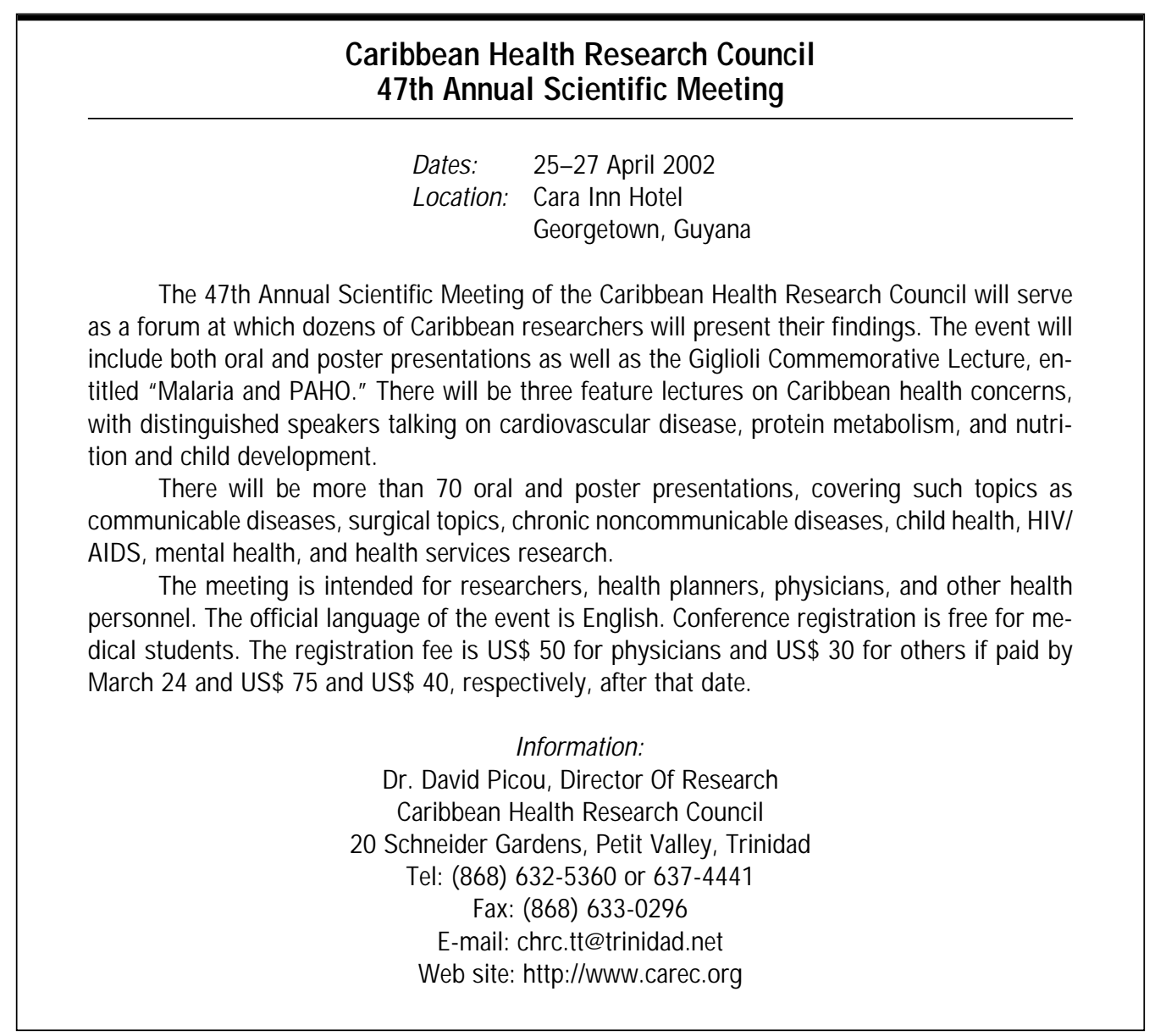

